\title{
Identificación de otros agentes infecciosos en casos sospechosos de síndrome cardiopulmonar por hantavirus
}

\author{
María Hinojosa Pla, Eliecer Villagra $C^{2}$, Judith Mora $\mathbf{R}^{2 b}$, \\ Liliana Maier ${ }^{1 b}$. \\ Identification of infectious agents \\ in cases suspicious of Hantavirus \\ infection but with negative serology
}

Background: The diagnosis of acute respiratory illness caused by Hantavirus is based in the rapid and progressive clinical course, epidemiological background and the serological confirmation of the virus. When the presence of the virus is not confirmed a differential diagnosis must be made with other infections. Between 1999 and 2001, the Chilean Public Health Institute received 1063 blood samples from patients with a suspicious clinical picture, to study the presence of Andes strain of Hantavirus. In 134 of these samples, the presence of the virus was confirmed. Aim: To study the presence of other infections in sera from patients with suspected Hantavirus acute respiratory illness but serologically negative for Hantavirus. Material and methods: A retrospective study of 98 serum samples, received at the National Public Health Institute, of patients with negative serology for Hantavirus. The presence of antibodies against influenza virus, Mycoplasma pneumoniae, Leptospira and Streptococcus pneumoniae was determined using Latex techniques. Results: Leptospira was detected in 23 patients, influenza virus in 13, Streptococcus pneumoniae in six and Mycoplasma pneumoniae in one case. Leptospira infections were confirmed in four cases by EUSA determination of IgM antibodies. Influenza virus infection was confirmed in three cases by Hemmaglutation Inhibition Assay. Conclusions: In suspected cases of Hantavirus acute respiratory infection, but with negative serology, other infectious agents such as Leptospira, influenza virus and Streptococcus pneumoniae, must be sought (Rev Méd Chile 2006; 134: 332-8).

(Key words: Hantavirus pulmonary syndrome; Leptospira; Orthomyxoviridae; Streptococcus pneumonia)

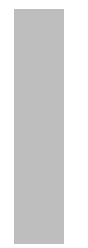

Recibido el 8 de enero, 2004. Aceptado el 15 de septiembre, 2005.

Fuente de financiamiento: Proyecto de investigación № 11, Escuela de Medicina Veterinaria, Universidad Santo Tomás.

${ }^{1}$ Facultad de Medicina Veterinaria, Universidad Santo Tomás

${ }^{2}$ Sección Virología, Instituto de Salud Pública de Chile

aAlumna tesista Escuela Medicina Veterinaria, Universidad Santo Tomás

${ }^{\mathrm{b}}$ Médico Veterinario

Correspondencia a: Dr. Eliecer Villagra Cornejo. Marathon 1000, Nuñoa, Santiago. Fax: 3507583. Fono: 3507448. E mail: evillagra@ispch.cl 
$\mathrm{E}^{1}$ Síndrome Cardiopulmonar por Hantavirus (SCPH) fue reconocido en 1993 en el suroeste de Estados Unidos, a raíz de un brote de muertes por distress respiratorio agudo ${ }^{1}$. A partir de este fecha se han notificado infecciones humanas causadas por este agente en diversos países de América, como Argentina, Canadá, Chile, Paraguay y Uruguay, con diferentes variedades del virus circulantes en estos países. En nuestro país se presenta sólo la variedad Andes, siendo su principal reservorio el Oligoryzomys longicaudatus, cuyo hábitat se extiende desde la III a la XI región ${ }^{2}$. En 1995 se identificó en Chile esta enfermedad por primera vez en un paciente de Cochamó, X región ${ }^{3}$. Hasta el año 2004 se han notificado alrededor de 380 casos confirmados de infección por Hantavirus, desde la V a la XI región del país ${ }^{4}$.

De acuerdo a la definición del Ministerio de Salud los casos sospechosos de SCPH corresponden a "todos aquellos individuos que presenten un cuadro febril con temperatura superior a $38,3^{\circ} \mathrm{C}$, con mialgias, cefaleas, acompañado o no de síntomas gastrointestinales, que presenta una radiografía de tórax con infiltrado intersticial uni o bilateral o un hemograma con: trombocitopenia, recuento de glóbulos blancos con desviación a la izquierda, inmunoblastos mayor a 10\% (linfocitos atípicos) y/o hemoconcentración y que además, tiene el antecedente de situaciones de riesgo como la exposición a roedores silvestres dentro de las 6 semanas previas al inicio de los síntomas o cuando presenta un cuadro de distress respiratorio (SDRA) sin causa aparente, que aparece en una persona previamente sana $\mathrm{O}$ todo cuadro respiratorio inexplicable, con resultado de muerte y cuya autopsia demuestre un edema pulmonar no cardiogénico sin una causa específica e identificable por las técnicas rutinarias de laboratorio" ${ }^{4}$.

El diagnóstico está basado tanto en su presentación clínica y rápida progresión como en los antecedentes epidemiológicos del paciente; sin embargo, existen otros agentes infecciosos, tales como: Leptospira sp, virus influenza, Mycoplasma pneumoniae, Streptococcus pneumoniae, entre otros, cuyas enfermedades se manifiestan en forma similar, siendo necesario realizar el diagnóstico diferencial ${ }^{5-9}$.
Entre los años 1999 y 2001, el Laboratorio de Hantavirus del Instituto de Salud pública (ISP) recibió un total de 1.063 muestras para estudio de infección por virus Andes provenientes de casos sospechosos, contactos y pacientes con distress respiratorio de otras etiologías. En 134 casos se confirmó la infección por hantavirus.

Este trabajo busca pesquisar la presencia de otros agentes infecciosos en aquellos pacientes que desarrollaron un SCPH, pero que no fue producido por virus Andes y adicionalmente caracterizar dichos pacientes.

\section{Material y Métodos}

En este estudio se analizaron en forma retrospectiva sueros de 98 pacientes, con diagnóstico de SCPH, provenientes en todo el país, entre los años 1999 y 2001, incluyéndose en el análisis las muestras de aquellos pacientes en que resultó negativa la detección de anticuerpos IgM para antígeno de virus Andes. Utilizándose para este estudio una técnica de ensayo inmunoenzimático (ELISA) efectuado en el Instituto de Salud Pública mediante técnica de referencia transferida por Center for Diseases Control (CDC). Se excluyeron de este estudio todos los pacientes con patologías previamente diagnosticadas crónicas, tales como: diabetes mellitus, procesos oncológicos, adicciones, edades extremas y pacientes con cuadros de inmunosupresión.

Las muestras fueron sometidas a técnicas de tamizaje tipo látex, para detectar patologías cuya sintomatología respiratoria aguda fuera similar al SCPH. Dentro de las patologías con estas características clínicas se escogieron para este estudio, las producidas por: virus influenza A y B, Leptospira sp, Mycoplasma pneumoniae y Streptococcus pneumoniae.

La técnica de látex -como toda técnica de tamizaje- puede tener resultados falsos positivos, por lo que fue necesario confirmar los resultados positivos tanta para Leptospira sp. como para virus influenza9. En el caso de Streptococcus pneumoniae y Mycoplasma pneumoniae, no se efectuó otra técnica confirmatoria, dado que se disponía sólo de una muestra de suero, que para un estudio bacteriológico no resulta la más adecuada. 


\section{Resultados}

Análisis de la población seleccionada para el estudio. Al clasificar la población seleccionada por sexo, se observó que $67,3 \%$ de los casos era de sexo masculino. La edad de la población en estudio comprendió un rango que fluctuó entre 3 y 90 años, con una edad promedio de 44,2 años.

Los casos predominaron entre los meses de octubre a abril, lo que corresponde a primavera y verano.

En relación a los factores de riesgo, en 80 de 98 casos se dispuso de la información correspondiente observándose un predominio de pacientes identificados como habitantes rurales, seguido de trabajadores agrícolas o forestales y excursionistas de localidades rurales (Figura 1).

Se clasificó la condición de salud de los pacientes al ingreso al centro hospitalario respectivo y al efectuar la notificación del caso sospechoso al Servicio de Salud correspondiente. Así se determinó que $75,5 \%$ de los 94 casos con información disponible, ingresó en estado grave o fallecido al centro asistencial (60,6\% grave y $14,9 \%$ fallecido), en comparación a $24,5 \%$ que no presentaron signos de gravedad.
De los casos graves, posteriormente fallecieron otros 7 pacientes, sumando un total de 21 fallecidos sobre 94 casos estudiados (22,34\%).

En la Tabla 1 se aprecia la mortalidad de los casos estudiados de acuerdo a los días de evolución del cuadro clínico, observándose que 4 casos evolucionaron muy rápidamente, con resultado de muerte dentro de las primeras $24 \mathrm{~h}$. Dentro de los primeros 5 días, un total de 11 individuos habían fallecido.

En la Tabla 2 se comparó el día de inicio de síntomas con el día de la toma de muestra para estudio de hantavirus, encontrándose $13,7 \%$ de los casos con 24 h de evolución y $52,1 \%$ recolectadas durante los primeros 5 días.

Analizando los síntomas encontrados en cada uno de los casos, el estudio demostró que todos presentaron compromiso respiratorio y $97,8 \%$ presentó una imagen radiográfica de tórax con evidencia de infiltrado intersticial. Fiebre se presentó en $82,6 \%$ de los casos, cefalea en $42,8 \%$, mialgias en $46,9 \%$ y síntomas gastrointestinales en $31,6 \%$ de los pacientes.

Los exámenes de laboratorio demostraron que $77,94 \%$ de los pacientes tuvo leucocitosis, $42,64 \%$ presentó trombocitopenia, $19,11 \%$ hematocrito

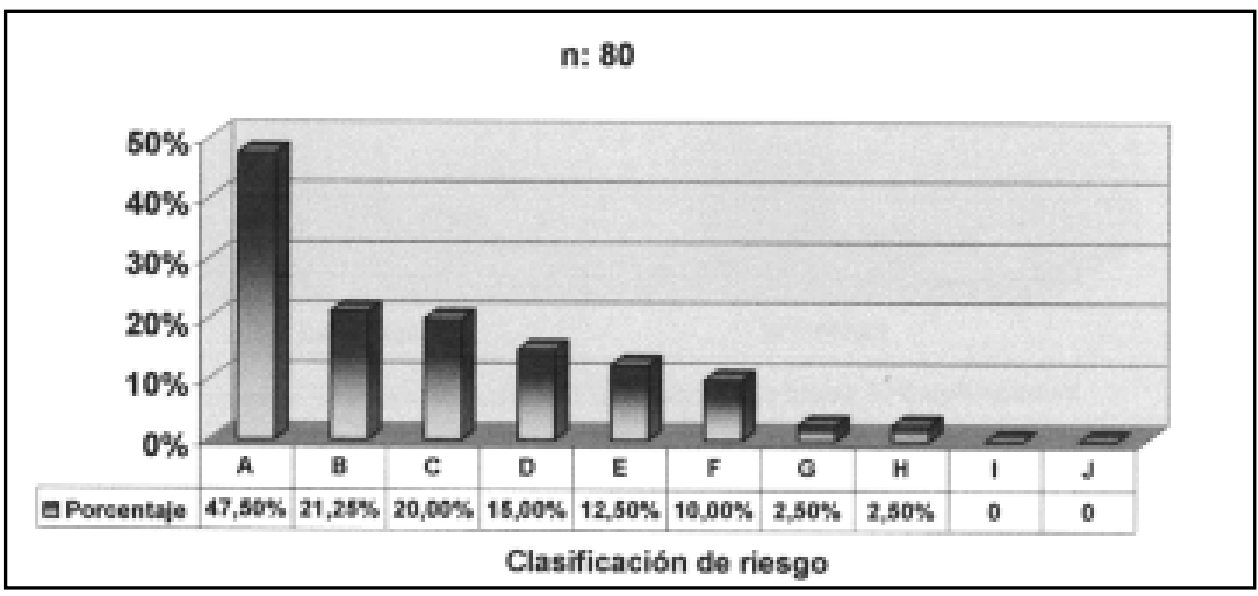

Figura 1. Distribución de factores de riesgo en casos sospechosos entre los años 1999-2001. Factores de riesgos asociados en la población estudiada según Formularios de Notificación:

A. Ruralidad, B. Trabajador agrícola-forestal, C. Excursionista de sectores rurales, D. Otro riesgo no señalado en esta tabla, E. Sin antecedentes de riesgo, F. Contacto con fuentes conocidas de contagio, G. Consumo o presencia de alimentos ró́dos, H. Contacto con caso sospechoso, I. Contacto con sangre y/o fluidos de personas infectadas y J. Herida por mordedura de ratón. 
Tabla 1. D istribución de la mortalidad en 21 casos estudiados de acuerdo a los días de evolución del cuadro clínico

\begin{tabular}{|lrrc|}
\hline Día & $\begin{array}{c}\text { № } \\
\text { casos }\end{array}$ & $\begin{array}{r}\text { Porcentaje } \\
\%\end{array}$ & $\begin{array}{c}\text { Porcentaje } \\
\text { acumulado }\end{array}$ \\
\hline 1 & 4 & 19,0 & 19,0 \\
2 & 1 & 4,8 & 23,8 \\
3 & 2 & 9,5 & 33,3 \\
4 & 3 & 14,3 & 47,6 \\
5 & 1 & 4,8 & 52,4 \\
6 & 2 & 9,5 & 61,9 \\
7 & 1 & 4,8 & 66,7 \\
8 & 1 & 4,8 & 71,5 \\
9 & 1 & 4,8 & 76,3 \\
11 & 2 & 9,5 & 85,8 \\
13 & 1 & 4,8 & 90,6 \\
14 & 1 & 4,8 & 95,4 \\
16 & 1 & 4,8 & 100,0 \\
Total & 21 & 100,0 & 100,0 \\
\hline
\end{tabular}

elevado y sólo 4,41\% presentó linfocitosis atípica, que hoy en día se sabe que corresponden a inmunoblastos.

Resultados de las pruebas diagnósticas. De los 98 casos de síndrome cardiopulmonar, en los que se descartó la infección por hantavirus, 23 casos $(23,5 \%)$ resultaron positivos por tamizaje a Leptospira sp. De ellos, 4 casos fueron confirmados positivos para Leptospira sp. En el caso de influenza, de los 98 casos, 13 resultaron positivos por tamizaje $(13,3 \%)$, de los cuales 3 fueron confirma-
Tabla 2. D istribución de los días de evolución al momento de la toma de la muestra para diagnóstico de $\mathrm{H}$ antavirus en 73 casos estudiados

\begin{tabular}{|lrrr|}
\hline $\begin{array}{l}\text { Días } \\
\text { evolución }\end{array}$ & $\begin{array}{c}\text { No } \\
\text { casos }\end{array}$ & $\begin{array}{c}\text { Porcentaje } \\
\%\end{array}$ & $\begin{array}{c}\text { Porcentaje } \\
\text { acumulado }\end{array}$ \\
\hline 1 & 10 & 13,7 & 13,7 \\
2 & 7 & 9,6 & 23,3 \\
3 & 9 & 12,3 & 35,6 \\
4 & 8 & 11,0 & 46,6 \\
5 & 4 & 5,5 & 52,1 \\
6 & 5 & 6,8 & 58,9 \\
7 & 11 & 15,1 & 74,0 \\
8 & 3 & 4,1 & 78,1 \\
9 & 3 & 4,1 & 82,2 \\
10 & 2 & 2,7 & 84,9 \\
11 & 4 & 5,5 & 90,4 \\
12 & 2 & 2,7 & 93,1 \\
14 & 2 & 2,7 & 95,8 \\
19 & 2 & 2,7 & 98,5 \\
23 & 1 & 1,4 & 100,0 \\
Total & 73 & 100,0 & 100,0 \\
& & & \\
\hline
\end{tabular}

dos como: 1 influenza A (H3N2) y 2 influenza B (Figura 2 y 3 ).

En el caso de neumococo, de las 98 muestras en estudio, 6 resultaron positivas por tamizaje (6,1\%). Para el caso de los micoplasmas, sólo 1 resultó positiva con bajo título por la prueba de látex $(1,02 \%)$. En estos casos no se dispuso de técnicas confirmatorias para identificar los agentes etiológicos.

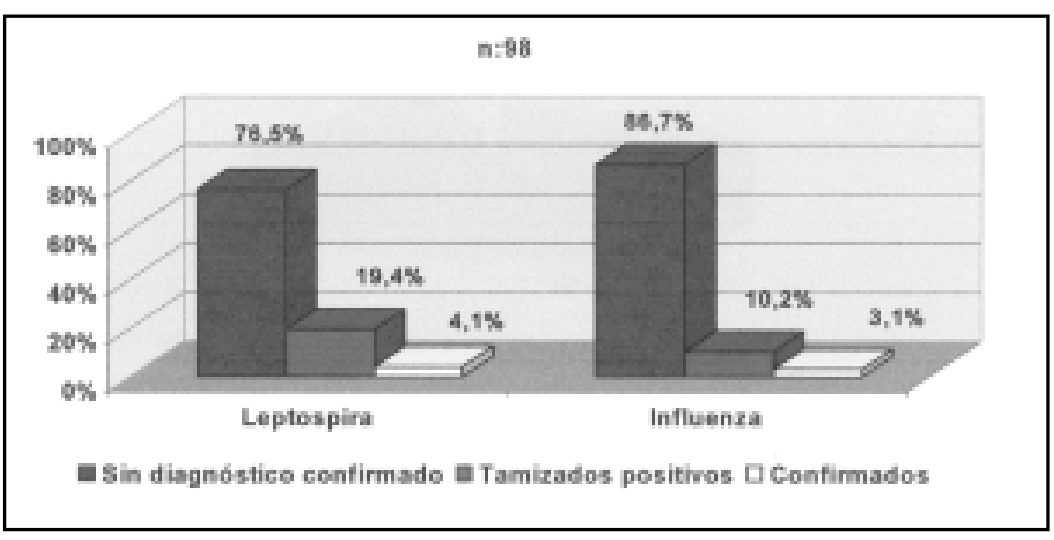

Figura 2. Resultados del diagnóstico de tamizaje y confirmados de Leptospira sp. e influenza, en 98 casos sospechosos de S.C.P entre los años 1999-2001. 


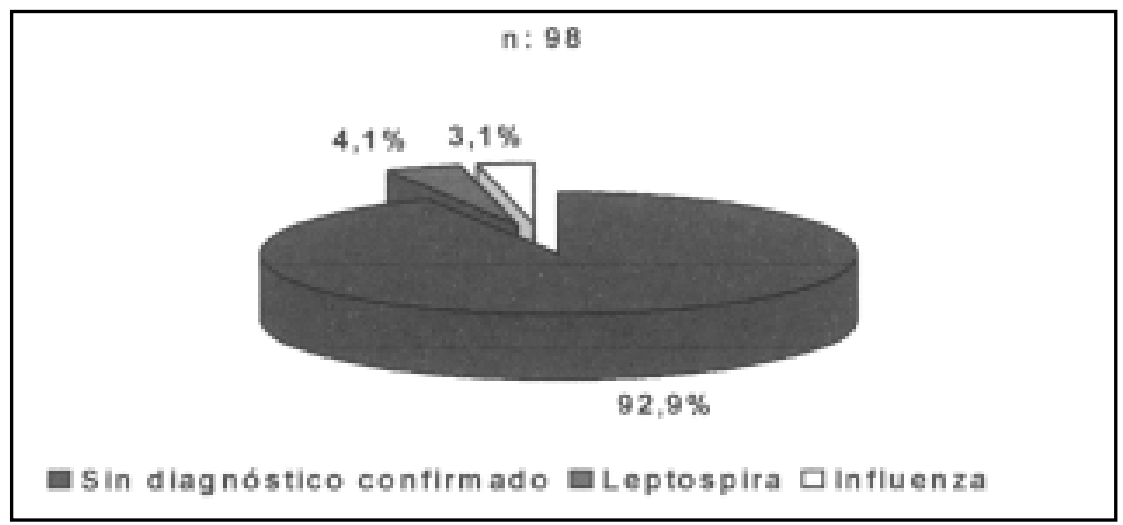

Figura 3. Agentes infecciosos confirmados en casos sospechosos de S.C.P negativos a hantavirus entre los años 1999-2001.

En las Tablas 3 y 4 se muestra un resumen de los antecedentes recopilados a partir de los formularios de notificación de cada paciente estudiado, bajo sospecha de infección por virus Andes y que posteriormente fueron descartados.

\section{DiSCUSIÓN}

Todos los pacientes seleccionados presentaron síndrome de distress respiratorio agudo con edema pulmonar y correspondieron principalmente a individuos del sexo masculino, de edades laboralmente activas entre 16 y 70 años, de origen predominantemente rural y que se presentaron entre los meses de octubre y abril, temporada de mayor actividad agrícola, lo que coincide con el perfil epidemiológico de los infectados por hantavirus ${ }^{10}$.

Al considerar la evolución clínica de los casos se encontró que $60,6 \%$ de los pacientes ingresaron en estado de gravedad y $15 \%$ fallecieron dentro de las $24 \mathrm{~h}$ de ingreso al recinto hospitalario.

La mortalidad general de $21,4 \%$ en la población estudiada es comparable a la detectada en los casos de SCPH del año 2002 cuya etiología fue confirmada para virus Andes ${ }^{11}$, lo que indica que hay otras enfermedades infecciosas agudas que deben ser consideradas como agentes etiológicos

Tabla 3. Antecedentes de los casos confirmados de leptospirosis entre los años 1999-2001

\begin{tabular}{|c|c|c|c|c|}
\hline & Caso 1 & caso 2 & Caso 3 & Caso 4 \\
\hline $\begin{array}{l}\text { Mes de } \\
\text { notificación }\end{array}$ & noviembre & diciembre & febrero & febrero \\
\hline Edad & 35 años & 57 años & 34 años & 40 años \\
\hline Sexo & femenino & masculino & femenino & femenino \\
\hline $\begin{array}{l}\text { Factor de } \\
\text { riesgo }\end{array}$ & $\begin{array}{l}\text { riesgo no } \\
\text { especificado }\end{array}$ & $\begin{array}{l}\text { ruralidad y } \\
\text { trabajador } \\
\text { agrícola }\end{array}$ & $\begin{array}{l}\text { sin } \\
\text { antecedentes }\end{array}$ & $\begin{array}{l}\text { excursionista } \\
\text { de localidades } \\
\text { rurales }\end{array}$ \\
\hline $\begin{array}{l}\text { Estado de } \\
\text { salud }\end{array}$ & no grave & fallecido & no grave & grave \\
\hline $\begin{array}{l}\text { Día de toma de } \\
\text { la muestra }\end{array}$ & séptimo día & duodécimo día & primer día & séptimo día \\
\hline
\end{tabular}


Tabla 4. Antecedentes de los casos confirmados de influenza entre los años 1999-2001

\begin{tabular}{|llll|}
\hline & Caso 1 & Caso 2 & Caso 3 \\
\hline Mes de notificación & diciembre & noviembre & enero \\
Edad & 71 años & 41 años & 74 años \\
Sexo & masculino & masculino & masculino \\
Factor de riesgo & sin antecedentes & ruralidad, & sin antecedentes \\
& & trabajador agrícola & \\
Estado de salud & grave & gexcursionista & \\
Día de toma de la muestra & primer día & tercer día & grave \\
& & & décimo día \\
\hline
\end{tabular}

fatales, especialmente en el caso de la leptospirosis y el neumococo, agentes que cuentan con tratamiento antibiótico efectivo.

De esos 21 fallecidos, se confirmó un caso de Leptospirosis. Se trató de un paciente de sexo masculino, de 57 años, agricultor de la quinta región, que se enfermó abruptamente y falleció al cabo de 2 semanas. El diagnóstico fue solicitado a los 12 días de evolución lo que indica que en este caso no se consideró el diagnóstico diferencial con leptospirosis.

Al igual que hantavirus, leptospira sp puede llegar al hombre a través del contacto con orina o tejidos de animales infectados, inclusive también penetrar en el organismo por heridas o abrasiones en la piel, conjuntiva, otras mucosas o por inhalación de gotitas o aerosoles que las conten$\operatorname{gan}^{12}$. En cuanto a la sintomatología clínica, la forma anictérica de la enfermedad es la más frecuente y su descripción clínica es parecida a la influenza u otras enfermedades febriles agudas ${ }^{13}$. Se señala en numerosas publicaciones que en países tropicales y subtropicales, es necesario realizar el diagnóstico diferencial con hantavirus entre otras enfermedades ${ }^{14}$.

El diagnóstico serológico de leptospirosis requiere que la toma de la muestra sea realizada después de 5 a 6 días de evolución para que puedan ser detectadas las IgM. En este estudio se observa que en 53 de los 96 casos estudiados, las muestras se tomaron antes de 5 días de evolución del cuadro clínico, por lo que es recomendable que durante el seguimiento del paciente se considere la toma de una segunda muestra seroló- gica, con el fin de disminuir el probable alto porcentaje de casos de leptospirosis subdiagnosticados en el presente estudio.

En el caso de influenza se requiere una muestra de aspirado nasofaríngeo para diagnóstico rápido de la etiología y de una muestra para realizar el diagnóstico serológico.

Debido a que Streptococcus pneumoniae es un patógeno que debiera ser considerado en el diagnóstico diferencial, es necesario tomar una muestra para hemocultivo.

Respecto a los parámetros de laboratorio hematológico, lo que se observó con mayor frecuencia fue leucocitosis, indicando que se trataba de cuadros predominantemente infecciosos. Por otra parte la trombocitopenia, también fue importante reafirmando el carácter de gravedad de los casos y que también corresponde a una característica observada en muchos casos de leptospirosis. Sin embargo, el aumento del hematocrito, frecuente en la infección por hantavirus se observó en menos de $20 \%$ de los casos estudiados.

Una diferencia significativa se observó en el bajo porcentaje de inmunoblastos encontrados en este estudio, apoyando su presencia como indicador de infección por hantavirus ${ }^{15}$.

Es importante destacar la necesidad de continuar el estudio del diagnóstico diferencial de los pacientes sospechosos de infección por hantavirus, puesto que en el análisis realizado sólo se determinó la etiología en 1 de los 21 fallecidos.

Como conclusión, se puede afirmar que existen cuadros clínicos semejantes a SCPH, que coinciden con la epidemiología de la infección por 
virus Andes, con una mortalidad de $21,4 \%$ y que tienen algunos elementos de laboratorio diferentes que son importantes de destacar: sólo 19\% presentó hematocrito elevado, fenómeno siempre presente en un paciente con distress respiratorio y sólo 4,4\% de inmunoblastos en la fase aguda de la enfermedad. Y finalmente que entre todos los pacientes fallecidos sólo en uno de los casos se logró llegar al diagnóstico etiológico de la enfermedad como causa de muerte.

Lo anterior plantea la necesidad de continuar estudios prospectivos, a fin de investigar otras

\section{REFERENCIAS}

1. Nichol S, Spiropoulou C, Morzunov S, Rollin P, Kiazer G, Feldmann H et al. Genetic identification of a hantavirus associated with an outbreak of acute respiratory illnes. Science 1993; 262: 914-7.

2. Murúa R. Etiología de los reservorios silvestres de hantavirus en Chile. Rev Chil Infect 1998; 15: 7983.

3. Toro J, Vega J, Khan A, Mills J, Padula P, Terry W ET AL. An outbreak of hantavirus pulmonary syndrome, Chile, 1997. Emerg Infect Dis 1998; 4: 667-702.

4. MINSAL. Boletín epidemiológico de hantavirus. Situación al 30 de diciembre de 2004. Ministerio de Salud de Chile.

5. BaUm S. Infecciones por micoplasma. En: Cecil, ed. Tratado de Medicina Interna de Wyngaarden, Smith, Bennett. Editorial Interamericana McGraw-Hill, 19ª edición 1994; vol 2: 1880-3.

6. McClain B. Leptospirosis. En: Cecil. Tratado de Medicina Interna de Wyngaarden, Smith, Bennett. Editorial Interamericana McGraw-Hill, 19a edición 1994; vol 2: 2067-9.

7. Chávez A, Mendoza C, Rojas C, Borghero A, Cerpa posibles causas que puedan explicar estos casos no aclarados, tomando en forma paralela otro tipo de muestra como tejidos, secreciones respiratorias y otros que permitan efectuar mayores análisis aumentando con ello la posibilidad de aclarar el diagnóstico y describir los agentes etiológicos primarios, causantes de este síndrome, sobre la base de un algoritmo, en caso de síndrome cardiopulmonar, que incluyan patologías tales como hantavirus, leptospirosis, influenza y neumococo como primera opción.

R. Experiencia clínica: infecciones invasoras por Streptococcus pneumoniae: impacto de la resistencia. Rev Chil Infect 2000; 17: 39-44.

8. Fica A. Profilaxis mediante la inmunización activa. Rev Chil Infect 2001; 18: 133-41.

9. Carballal G, Oubiña J. Virología Médica 1998. Editorial Ateneo, $3^{\mathrm{a}}$ Edición Capítulo 15: 289-90.

10. SChMAjjhon C, HJelle B. Hantavirus: A global disease problem. Emerg Infect Dis 1997; 3: 95-104.

11. MINSAL. Boletín epidemiológico de hantavirus. Situación al 20 de noviembre de 2002. Ministerio de Salud de Chile.

12. Muñoz L, VAlenzuela M. Caso clínico de Leptospirosis canina. Revista de la Sociedad de Médicos Veterinarios especialistas en pequeños animales (Mevepa). 1999; 13: 32-5.

13. Lomar AV, Diament D, Torres JR. Leptospirosis in Latin America. Infect Dis Clin North Am 2000; 14: 23-39.

14. Lewett PN. Leptospirosis. Clin Microbiol Rev 2001; 14: 296-326.

15. Romero C, Andresen M, Díaz O, Tomicic V, BarahoNA F ET AL. Síndrome cardiopulmonar por Hantavirus: utilidad de la monitorización con el sistema PiCCO. Rev Méd Chile 2003; 131: 1173-8.

\section{Agradecimientos}

Los autores desean agradecer a la Dra. Liliana Urra, por sus análisis confirmatorios de leptospirosis, y al Dr. Rodrigo Fasce, por el análisis de influenza por la técnica de inhibición de la hemoaglutinación, ambos del Instituto de Salud Pública de Chile. 